Check for updates

Cite this: Mater. Adv., 2020 1,3398

Received 9th October 2020,

Accepted 9th November 2020

DOI: $10.1039 / \mathrm{d} 0 \mathrm{ma00778a}$

rsc.li/materials-advances

\section{Decoupled ion mobility in nano-confined ionic plastic crystal $\dagger$}

\author{
Haijin Zhu, (D)*ab Aleksandra Grzelak, ${ }^{a b}$ Ruhamah Yunis, ID c Jaime Martín (D) de and \\ Maria Forsyth iD abd
}

\begin{abstract}
Nanoconfined ions have dramatically different local environments compared to the bulk, which profoundly affects the ion solvation and transport properties taking place in the confined space. Herein, we investigate the rotational and translation mobility of both cation and anions of an OIPC (diethyl)(methyl)(isobutyl)phosphonium hexafluorophosphate) confined in 40 and $180 \mathrm{~nm}$ straight-through $\mathrm{Al}_{2} \mathrm{O}_{3}$ pores. The results revealed that the nanoconfined OIPC exhibit 44 times higher ionic conductivity than the bulk material at $30{ }^{\circ} \mathrm{C}$. This enhancement is attributed to both the reduced tortuosity and the increased population of mobile species. More interestingly, the $\mathrm{Al}_{2} \mathrm{O}_{3}$ nanochannels were found to selectively enhance the rotation and translational motion of $\left[\mathrm{P}_{122 i 4}\right]$ cation at elevated temperatures, whilst leaving that of the $\left[\mathrm{PF}_{6}\right]$ anion less affected.
\end{abstract}

\section{Introduction}

The ever increasing safety and stability concerns associated with organic solvent based liquid electrolytes have encouraged the use of all-solid materials for electrochemical device applications. Despite the tremendous efforts in both industry and academia dedicated to developing solid electrolytes using inorganic salts, ceramics, polymers, and their composites, most of these materials suffer from a significantly lower conductivity compared to the liquid electrolytes. A fully hydrated Nafion ${ }^{\circledR}$, for example, can easily achieve conductivity of $>10^{-1} \mathrm{~S} \mathrm{~cm}^{-1},{ }^{1}$ whereas hardly any polymer electrolyte can achieve a bulk conductivity (without solvent) of the order of $10^{-3} \mathrm{~S} \mathrm{~cm}^{-1}$ at room temperature. ${ }^{2}$

Organic ionic plastic crystals (OIPCs) represent a unique family of solid materials with demonstrated applications as solid-state electrolytes for lithium and sodium ion batteries, ${ }^{3,4}$ fuel cells, ${ }^{5,6}$ and dye-sensitized solar cells. ${ }^{7-9}$ OIPCs typically consist of a bulky organic cation coupled with a highly symmetric anion. ${ }^{10}$ The relatively large size and high symmetry of

\footnotetext{
${ }^{a}$ Institute for Frontier Materials, Deakin University, Geelong, VIC 3216, Australia. E-mail:h.zhu@deakin.edu.au

${ }^{b}$ ARC Centre of Excellence for Electromaterials Science, Deakin University, 221 Burwood Highway, Burwood, Victoria 3125, Australia

${ }^{c}$ Centre for Materials Science, School of Chemistry and Physics, Queensland University of Technology, 2 George Street, Brisbane, Queensland, 4000, Australia

${ }^{d}$ POLYMAT and Polymer Science and Technology Department, Faculty of Chemistry, University of the Basque Country UPV/EHU, Manuel de Lardizabal 3,

20018 Donostia-San Sebastian, Spain

${ }^{e}$ Ikerbasque, Basque Foundation for Science, 48013 Bilbao, Spain

$\dagger$ Electronic supplementary information (ESI) available. See DOI: 10.1039/ d0ma00778a
}

ions give rise to a balance in interionic interactions, which are strong enough to maintain the long-range crystalline lattice structure, yet weak enough to allow local molecular rotation and tumbling in their solid plastic crystal phases. ${ }^{10}$ These fast local molecular motions, as well as the associated structural disorder, not only can induce fast-ion transport of doped target ions such as $\mathrm{Li}^{+},{ }^{4} \mathrm{Na}^{+},{ }^{11} \mathrm{H}^{+},{ }^{12}$ but also confer the material with good mechanical ductility, allowing a tight and durable contact between electrolyte and the electrodes. ${ }^{13}$

Since the very first ammonium-based OIPCs reported in the 1980s by Nakamura and coworkers, ${ }^{14,15}$ many new OIPC families have been discovered and applied in various devices, including the first demonstration of OIPC in $\mathrm{Na}$ batteries recently. ${ }^{11,16}$ Nevertheless, the ionic conductivities of bulk OIPCs in their plastic crystal phases are moderate, roughly in the range of $10^{-6}-10^{-4} \mathrm{~S} \mathrm{~cm}^{-1}$ depending on sample preparation and thermal history. These values are still typically 1-2 order of magnitudes lower than their ionic liquid relatives. On the other hand, the OIPC itself is often soft and waxy, thus a support is required to provide structural and mechanical integrity. For these considerations, various polymer matrices including PVDF ${ }^{17,18} \mathrm{PEO}^{19}{ }^{19}$ Poly(ionic liquid)s, ${ }^{20,21}$ as well as various inorganic fillers, e.g., $\mathrm{TiO}_{2},{ }^{22} \mathrm{Al}_{2} \mathrm{O}_{3},{ }^{23} \mathrm{SiO}_{2}{ }^{23,24}$ have been introduced, aiming to increase mechanical strength and enhance the ionic conductivity simultaneously. Recent studies have shown that adding a second phase does not only provide a mechanical support, but also brings in an unique interface which had synergistic effect in boosting their ionic conductivities. ${ }^{25}$ Wang et al., demonstrated that co-electrospun OIPC composites comprised of between 5 and 20 wt\% of $\left[\mathrm{C}_{2} \mathrm{mpyr}\right]\left[\mathrm{BF}_{4}\right]$ combined with PVDF, had room temperature conductivity that was enhanced by 7 -fold (compared to neat OIPC). ${ }^{25}$ 
The key mechanism for this conductivity enhancement was the disruption of crystallization and enhanced ion dynamics in the OIPC phase due to the ion-dipole interactions between OIPC and PVDF molecules. A maximum conductivity was found at $15 \mathrm{wt} \%$ OIPC loading, and further increasing OIPC leads to a sharp decrease in conductivity. This is attributed to the fact that the ion conduction took place only along a thin layer of (percolated) amorphous OIPC/PVDF interphase. At high OIPC loadings there will be more ordered OIPC present and thus the ion conduction will be 'blocked' by this crystalline OIPC phase. This is strongly supported by the DSC heating thermograms where no first-order transition was observed for the sample of 5-15 wt\% OIPC loading, whereas the $20 \mathrm{wt} \%$ loading clearly showed peaks corresponding to crystalline OIPC phase.

An alternative strategy to increase the ionic conductivity of OIPCs is through the addition of nanoparticles. Adding nanosized $\mathrm{TiO}_{2}{ }^{22}$ or $\mathrm{SiO}_{2}{ }^{26}$ into $\left[\mathrm{C}_{2} \mathrm{mpyr}\right][\mathrm{TFSI}]$ was found to increase ionic conductivity by approximately 1-2 orders of magnitude. An optimum concentration of $10 \mathrm{wt} \%$ was observed for these inorganic nanoparticle/OIPCs composites, which is similar to the optimum OIPC loading of $15 \mathrm{wt} \%$ for $\left[\mathrm{C}_{2} \mathrm{mpyr}\right]\left[\mathrm{BF}_{4}\right] / \mathrm{PVDF}$ composites observed by Wang et al. ${ }^{25}$ These results therefore suggested a percolation-dominated ion conduction mechanism in these composites, although the positron annihilation lifetime spectroscopy (PALS) results suggested that the percolated mobile phase may not be merely a thin layer of OIPC molecules on the particle surfaces, but instead, an interface induced disorder region that extends to the bulk OIPC with a length scale of about $10 \mathrm{~nm}^{26}$

In this study, we incorporate a well-known OIPC (diethyl)(methyl) (isobutyl)phosphonium hexafluorophosphate $\left.\left(\left[\mathrm{P}_{122 \mathrm{i}}\right]\left[\mathrm{PF}_{6}\right]\right)\right)^{4,27-29}$ into hexagonal aligned straight-through $\mathrm{Al}_{2} \mathrm{O}_{3}$ nano pores with diameters of 40 and $180 \mathrm{~nm}$. It is anticipated that the nanometer pore size provides a large surface-to-bulk ratio and boosts NMR signals from the interphase region, allowing us to probe ion transport phenomenon within the interphases. Secondly, using straight-through pores will also give unity (smallest) tortuosity for ion transport. The effects of nanoconfinement on the rotational and translational mobility of both cation and anion are investigated. We found that the cation and anion exhibit decoupled ion motilities, and each solid-solid phase transition is associated primarily with the onset of motions of either cation or anion, not both. The cation and anion transport behaviours within the $40 \mathrm{~nm}$ channels are compared to that in the bulk $\left[\mathrm{P}_{122 \mathrm{i} 4}\right]\left[\mathrm{PF}_{6}\right]$ material, with particular emphasis on the effect of tortuosity and surface interactions between ionic species and the $\mathrm{Al}_{2} \mathrm{O}_{3}$ walls.

\section{Experimental}

\subsection{Sample preparation}

The mesoporous $\mathrm{Al}_{2} \mathrm{O}_{3}$ membranes with 40 and $80 \mathrm{~nm}$ throughpores were purchased from Smartmembranes GmbH Germany. The $\left[\mathrm{P}_{122 \mathrm{i}}\right]\left[\mathrm{PF}_{6}\right]$ OIPC was synthesized following the procedures described elsewhere. ${ }^{30}$ The OIPC was purified by crystallization from a mixture of ethyl acetate and methanol. The molecular structure is shown in Fig. 1, and the chemistry of the synthesized material and the purity were confirmed by mass spectroscopy, ${ }^{1} \mathrm{H},{ }^{13} \mathrm{C},{ }^{19} \mathrm{~F}$ NMR and ICP-MS (residual potassium contents). The cation and anion were identified in the mass spectrum as shown in Fig. 1. The composite material was prepared by melting the $\left[\mathrm{P}_{122 \mathrm{i} 4}\right]\left[\mathrm{PF}_{6}\right]$ into nanopores on a $160{ }^{\circ} \mathrm{C}$ hot stage. The excess of OIPC material was wiped out carefully with a doctor blade. Specifications of the membranes and the composites are summarized in Table 1. As shown, the porosity for the 40 and $180 \mathrm{~nm}$ membranes, as calculated by $\frac{V_{\text {pore }}}{V_{\text {membrane }}}$, are $8 \mathrm{vol} \%$ and $11 \mathrm{vol} \%$, respectively. The volume percentages of the experimentally loaded OIPC, which were calculated from the weight difference between the empty and loaded membranes, are $10 \mathrm{vol} \%$ and 14 vol\% respectively, slightly larger than the porosity of the membranes. This suggest an excess of OIPC material remained on the membrane surface, which is beneficial for a good contact with electrodes during conductivity measurements. For the ease of discussion, the neat $\left[\mathrm{P}_{122 \mathrm{i} 4}\right]\left[\mathrm{PF}_{6}\right]$ and the composites with $40 \mathrm{~nm}$ and $180 \mathrm{~nm}$ $\mathrm{Al}_{2} \mathrm{O}_{3}$ membranes will be referred as neat, $40 \mathrm{~nm}$ and $180 \mathrm{~nm}$ in the following text.

\subsection{Ionic conductivity, SEM and DSC characterisations}

Ionic conductivity was measured using a Solartron FRA 1296 -frequency response analyser, supported by Solartron software. The neat $\left[\mathrm{P}_{122 \mathrm{i} 4}\right]\left[\mathrm{PF}_{6}\right]$ was pressed into a pellet with thickness of $1 \mathrm{~mm}$ and diameter of $13 \mathrm{~mm}$. The neat OIPC pellet and composite membranes were placed between two stainless steel electrodes for conductivity measurements. Data were collected over a frequency range from $10 \mathrm{MHz}$ to $1 \mathrm{~Hz}$ using a signal voltage of $0.1 \mathrm{~V}$. A heating-cooling-heating temperature program was applied between $20-160{ }^{\circ} \mathrm{C}$ with a step of $10{ }^{\circ} \mathrm{C}$. The samples were left to equilibrate for $10 \mathrm{~min}$ at each temperature. A ZEISS SUPRA 55VP FEG scanning electron microscope was used to characterize the surface morphology. The acceleration voltage was $3 \mathrm{kV}$. The same samples after EIS measurements were coated with Au and then submitted for SEM examination. The DSC measurements were performed on a Netzsch DSC (214 polyma). About 6-10 mg of the samples was sealed in an aluminum sample pan in the glovebox. A three-step heating-cooling-heating program

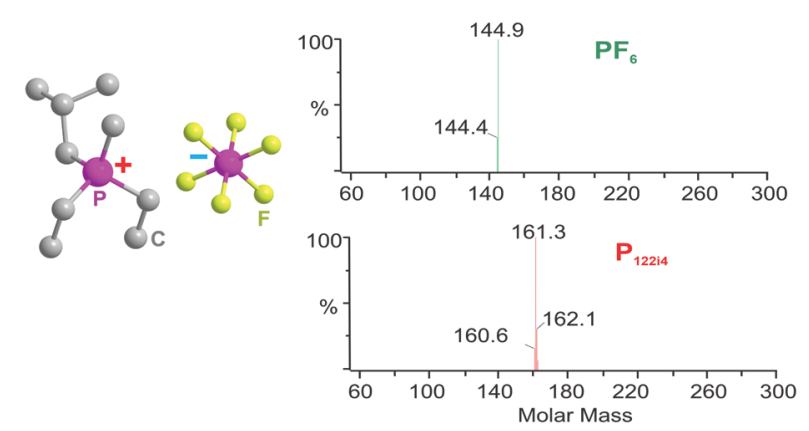

Fig. 1 Molecular structure and mass spectroscopy of the OIPC $\left[\mathrm{P}_{122 i 4}\right]\left[\mathrm{PF}_{6}\right]$. 
Table 1 Specification of the $\mathrm{Al}_{2} \mathrm{O}_{3}$ nanopores and composites

\begin{tabular}{llllll}
\hline $\begin{array}{l}\text { Pore } \\
\text { size }[\mathrm{nm}]\end{array}$ & $\begin{array}{l}\text { Pore } \\
\text { length }[\mu \mathrm{m}]\end{array}$ & $\begin{array}{l}\text { Pore } \\
\text { shape }\end{array}$ & $\begin{array}{l}\text { Pitch }^{a} \\
{[\mathrm{~nm}]}\end{array}$ & $\begin{array}{l}\text { Porosity } \\
\text { vol\% }\end{array}$ & $\begin{array}{l}\text { OIPC } \\
\text { loading }(\mathrm{mg})\end{array}$ \\
\hline 40 & 100 & Straight & 125 & 8 & $5.5 \pm 1$ \\
180 & 100 & Straight & 480 & 11 & $6.8 \pm 1.2$ \\
${ }^{a}$ Centre-to-centre distance. & & &
\end{tabular}

between temperature range of $-10-160{ }^{\circ} \mathrm{C}$ was applied, with a heating and cooling rate of $10{ }^{\circ} \mathrm{C} \mathrm{min}^{-1}$.

\subsection{Solid-state and pulse field gradient (PFG) NMR}

Static ${ }^{1} \mathrm{H},{ }^{19} \mathrm{~F}$ and ${ }^{31} \mathrm{P}$ NMR were performed on a $500 \mathrm{MHz}$ Bruker Avance III wide-bore solid state NMR spectrometer equipped with a $5 \mathrm{~mm}$ static double resonance probe. The alumina membrane sample was broken into small pieces and filled into a $4 \mathrm{~mm} \mathrm{ZrO}_{2}$ MAS rotor which was then sealed with a $\mathrm{ZrO}_{2}$ cap in an Argon filled glovebox. The sealed rotor was transferred out of the glovebox and inserted into the sample chamber of the static probe for static powder pattern NMR experiments. The same rotors were used later for PFG-NMR experiments, which were performed on a $300 \mathrm{MHz}$ Bruker Avance III widebore spectrometer equipped with a Diff50 diffusion probe. The maximum gradient strength of this probe is $29 \mathrm{~T} \mathrm{~m}^{-1}$. Diffusion time was varied between $8 \mathrm{~ms}$ and $400 \mathrm{~ms}$ in a $\log$ scale to probe the restricted diffusion behaviour. Sample temperature in the static NMR probe was calibrated with ${ }^{207} \mathrm{PbNO}_{3}$ using the method described in the literature. ${ }^{31}$ The Diff50 probe temperature was calculated using the chemical shift separation between the $\mathrm{OH}$ and $\mathrm{CH}_{3}$ groups of dry methanol. ${ }^{32}$

\section{Results and discussion}

\subsection{Morphology, thermal property and ion conduction}

Fig. 2a and b present typical SEM surface images of the $40 \mathrm{~nm}$ and $180 \mathrm{~nm}$ raw $\mathrm{Al}_{2} \mathrm{O}_{3}$ membranes. As shown, the hexagonally aligned nanopores are highly ordered with a narrow size distribution. The ionic conductivities of $40 \mathrm{~nm}$ and $180 \mathrm{~nm}$, as shown in Fig. 2c, are dramatically higher than the neat OIPC in phase II and III. This enhancement is particularly significant at lower temperatures. For example, the $40 \mathrm{~nm}$ pore confined OIPC shows conductivities of $4.5 \times 10^{-4}$ and $5.5 \times 10^{-6} \mathrm{~S} \mathrm{~cm}^{-1}$ at 120 and $30{ }^{\circ} \mathrm{C}$, respectively, which are 5 and 44 times that of the neat OIPC. It is interesting to note that, while a sharp nonlinear increase with temperature was observed upon phase III $\rightarrow$ II transition for neat OIPC, this behaviour was not as evident for the nanoconfined OIPCs, suggesting different ion conduction mechanisms. DSC traces of the neat OIPC show three distinct solid-solid transition peaks at about $25{ }^{\circ} \mathrm{C}, 72{ }^{\circ} \mathrm{C}$, $120{ }^{\circ} \mathrm{C}$, and a melting transition at $150{ }^{\circ} \mathrm{C}$ with a low entropy of fusion. These transitions are consistent with those reported previously. ${ }^{30}$ The $40 \mathrm{~nm}$ and $180 \mathrm{~nm}$ composites show very similar solid-solid transitions compared to the neat OIPC, except for the melting transition of $40 \mathrm{~nm}$ which was a bit broader. Note that the endothermic peaks in the DSC thermograms only
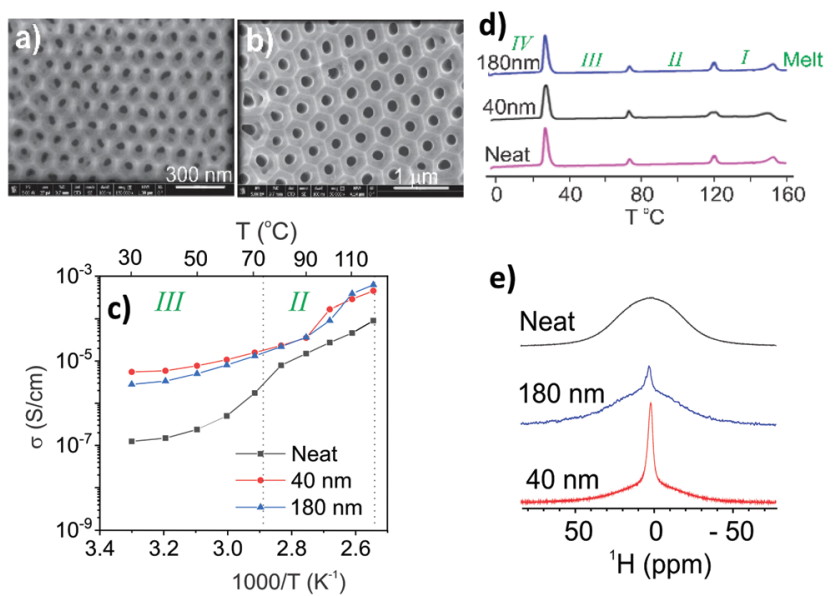

e)

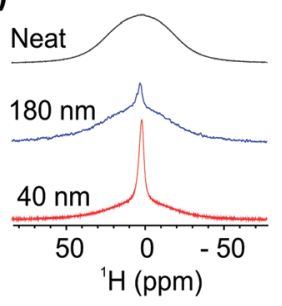

Fig. 2 Surface morphology, ionic conductivity and thermal behaviour of the nano confined OIPC. (a) and (b) are the SEM images of the aligned through-pores of the $40 \mathrm{~nm}$ and $180 \mathrm{~nm}$ raw membranes; (c) ionic conductivities and (d) DSC heat-flow rate signals for neat, $40 \mathrm{~nm}$ and $180 \mathrm{~nm}$ samples. A heating-cooling-heating temperature program was applied, and the second heating scans were reported for both conductivity and DSC measurements. The conductivities of $40 \mathrm{~nm}$ and $180 \mathrm{~nm}$ samples were normalized by the porosity of the membranes, which are 8 vol\% and 11 vol\%, respectively. (e) A comparison of static ${ }^{1} \mathrm{H}$ NMR spectra of neat OIPC, and that confined in 180 and $40 \mathrm{~nm} \mathrm{Al} \mathrm{O}_{3}$ nanopores at $20{ }^{\circ} \mathrm{C}$ (phase IV).

characterize first-order transitions which occur in the crystalline region, while the differences in the amorphous or defect region are not reflected in these DSC curves. Solid state NMR lineshape analysis, on the other hand, is an extremely valuable analytical tool for assessing the molecular dynamics in both crystalline and amorphous regions. ${ }^{15,33}$ Indeed, static ${ }^{1} \mathrm{H}$ NMR spectra in Fig. 2e suggest that the key factor that leads to higher conductivity of the nanoconfined OIPCs lies in the amorphous region in between the grain boundaries, which is consistent with previous MRI visualisation of an mobile amorphous grain boundary region with thickness of a few hundreds of nanometres. ${ }^{28,34}$ At $20{ }^{\circ} \mathrm{C}$ for example, neat OIPC shows a broad Gaussian line, with no sign of a narrow resonance observed. This agrees well with what has been reported in prior literature. ${ }^{30}$ The $180 \mathrm{~nm}$ sample clearly shows a sharp narrow line on top of a broad line, which hints at the existence of a fraction of mobile cation species. The $40 \mathrm{~nm}$ sample shows an even larger portion of these mobile cations, as indicated by the much stronger narrow line intensity.

\subsection{Tumbling and reorientation motion}

To further understand the effect of nanoconfinement on the local molecular motions of the OIPC ions, we performed a detailed multinuclear static NMR study on the $40 \mathrm{~nm}$ sample, which has a smaller pore size and consequently a larger surface area. An interesting feature of this OIPC is that both cation and anion contain a phosphorous nucleus which allows us to easily compare the line-narrowing behaviour between cation and anion in a single ${ }^{31} \mathrm{P}$ spectrum. Fig. 3a shows progressive line narrowing of both cation and anion in neat OIPC with temperature. However, a close examination of the line narrowing 
behaviour revealed that the cation and anion exhibit very different solid-phase transition dependency. Upon IV $\rightarrow$ III transition, the cation lineshape narrowed significantly, whereas the anion line showed nearly no change. Upon III $\rightarrow$ II transition, the anion line narrowed sharply, exhibiting a feature of $J$-coupling spitting which is typically observed for liquid samples. The cation line, on the other hand only showed a gradual narrowing with temperature. Then II $\rightarrow$ I transition is featured by a sudden decrease in cation linewidth, whereas the anion line remained essentially unchanged. The final melting transition at about $153{ }^{\circ} \mathrm{C}$ leads to a sudden extreme narrowing for both cation and anion lines. These results strongly suggest a highly decoupled cation and anion dynamics throughout the entire solid phases, and each of the solid-solid transitions are mainly associated with the onset of motions in either cation or anion, but not both. More specifically, the IV $\rightarrow$ III transition at $26{ }^{\circ} \mathrm{C}$, and II $\rightarrow$ I transition at $120{ }^{\circ} \mathrm{C}$ are associated with the cation, whereas the III $\rightarrow$ II transition at $72{ }^{\circ} \mathrm{C}$ is associated with the anion. Using this understanding, we may explain the extremely low phase III $\rightarrow$ II transition entropy of about $4 \mathrm{~J} \mathrm{~K}^{-1} \mathrm{~mol}^{-1}$, which might be related to the intrinsically high symmetry of the $\mathrm{PF}_{6}$ anion. The highly decoupled cation and anion dynamics is further confirmed by the static ${ }^{1} \mathrm{H}$ NMR in Fig. 4, and the ${ }^{19} \mathrm{~F}$ static NMR in Fig. 5. For the neat OIPC sample, a sharp ${ }^{1} \mathrm{H}$ line narrowing was observed over IV $\rightarrow$ III and II $\rightarrow$ I transitions, while a sharp ${ }^{19} \mathrm{~F}$ line narrowing was observed upon III $\rightarrow$ II transition.

The comparison of ${ }^{1} \mathrm{H}$ spectra in Fig. 4 revealed significantly different cation mobilities between the $40 \mathrm{~nm}$ and neat OIPC. More specifically, a significant narrow component, which represents the mobile species, is superimposed on the broader ${ }^{1} \mathrm{H}$ peak for the $40 \mathrm{~nm}$ sample, even at low temperatures (phase IV). However this narrow component was not observed for the neat sample till the temperature raised up to $40{ }^{\circ} \mathrm{C}$. With further increasing temperature, only a tiny fraction $(\sim 1 \mathrm{~mol} \%)$ of this species presented till the temperature reaches phase I. Interestingly, the ${ }^{19} \mathrm{~F}$ spectra in Fig. 5, shows similar anion

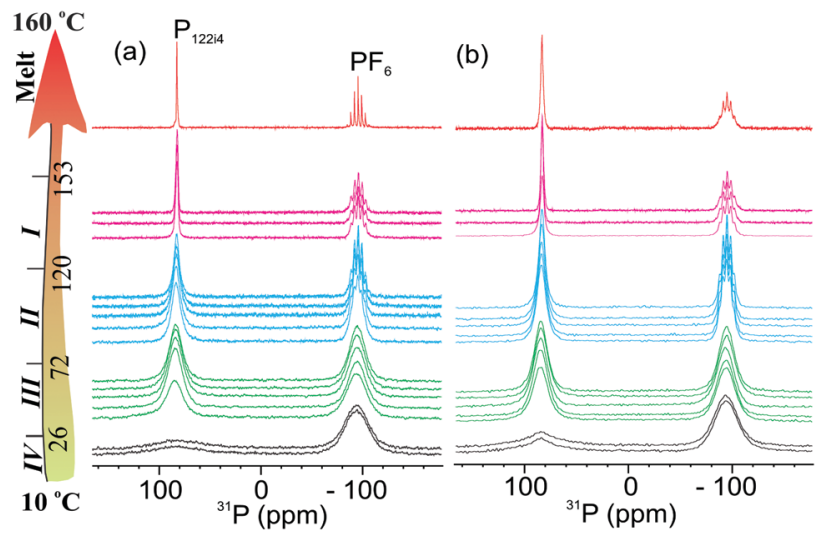

Fig. 3 Stack plots of the variable temperature static ${ }^{31} P$ NMR spectra of (a) neat OIPC and (b) the $40 \mathrm{~nm}$ sample, with separate peaks for cation and anion. The temperature was increased from 10 to $160{ }^{\circ} \mathrm{C}$ with a step of $10{ }^{\circ} \mathrm{C}$. The solid transition temperatures are indicated on the arrow at left-side, and spectra of each solid phase are grouped and coded with a different color.
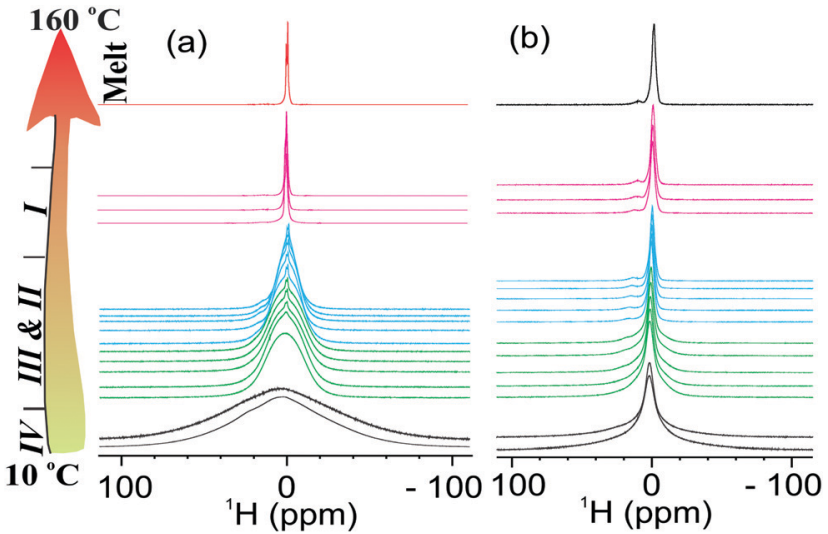

Fig. 4 Stack plots of the variable temperature static ${ }^{1} \mathrm{H}$ NMR spectra of cation in (a) neat OIPC and (b) $40 \mathrm{~nm}$ sample. The temperature was increased from 10 to $160{ }^{\circ} \mathrm{C}$ with a step of $10^{\circ} \mathrm{C}$.
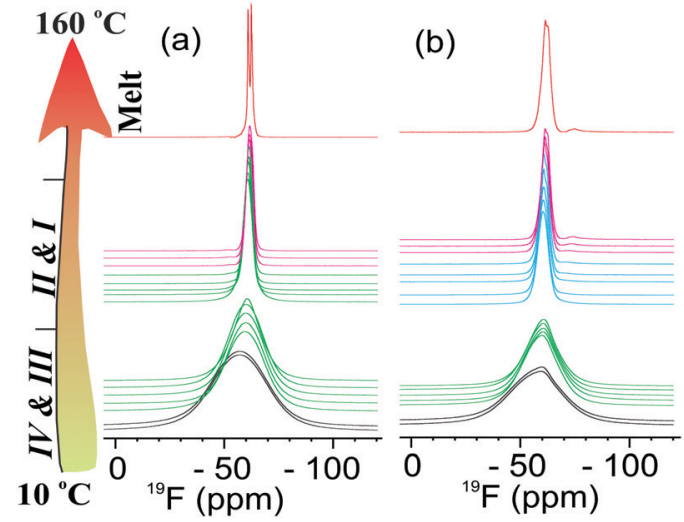

Fig. 5 Stack plots of the variable temperature static ${ }^{19} \mathrm{~F}$ NMR spectra of anion in (a) neat OIPC and (b) the $40 \mathrm{~nm}$ sample. The temperature was increased from 10 to $160{ }^{\circ} \mathrm{C}$ with a step of $10{ }^{\circ} \mathrm{C}$.

lineshapes in both samples in the studied temperature range. This indicates that the nanoconfinment affects the cation and anion mobility differently: the cation mobility is more enhanced relative to the anion mobility as a result of nanoconfinement. This is highly consistent with our diffusion measurements where we also observed enhancement in cation diffusion, whereas the anion diffusion coefficients were very close to the neat sample, as will be discussed in the following Section 3.3. At $160{ }^{\circ} \mathrm{C}$, both ${ }^{1} \mathrm{H}$ and ${ }^{19} \mathrm{~F}$ spectra of the $40 \mathrm{~nm}$ sample showed broader lines compared to the neat OIPC, indicating restricted ion motilities in the melt state.

\subsection{Translational motion}

Ion diffusion is a key process that determines the conductivity of the materials, and this process is known to behave differently in a confined space than the bulk. In an infinitely large space, the diffusion coefficient of a particle is independent of diffusion time. In a confined space, however, the wall collisions cause the diffusion coefficient to reduce. The observed diffusion coefficient is a function of diffusion time, and the function varies for different pore sizes and geometry. ${ }^{35}$ For isolated pores, the 
diffusion coefficients approach zero when $\sqrt{6 D_{0} t} \gg d$, where $D_{0}$ is the diffusion coefficient, $t$ is the diffusion time and $d$ is the characteristic length of the closed space. For interconnected pores, the diffusion coefficient decreases with increasing diffusion time and reaches an asymptotic value $D_{\infty}, D_{\infty}=D_{0} / \alpha,{ }^{36}$ where $\alpha$ is the tortuosity, $\alpha=1$ for a straight line and $\alpha=\infty$ for a circle. In an ideal one dimensional nanochannel where the $\sqrt{6 D_{0} t} \gg d$ ( $d$ is the diameter of the channel), the diffusion is fully restricted in two directions that are perpendicular to the length of the channel, and is free along the length direction. Powder averaging of the diffusion in randomly oriented ion channels would result in an apparent diffusion coefficient $D_{\text {app }}=\frac{1}{3} D_{0},{ }^{37}$ where $D_{0}$ is the diffusion coefficient we would obtain when all the channels are perfectly aligned with the direction of the magnetic field gradient.

As shown in Fig. 6(a) and (b), the diffusion coefficients of cation and anion in neat OIPC decrease with diffusion time, indicating a restricted diffusion within a confined space. This observation is consistent with the previous MRI study that showed the mobile ions are confined within a grain boundary phase with a characteristic thickness of a few hundreds of nanometres. ${ }^{34}$ The $\left[\mathrm{PF}_{6}\right]$ anion shows systematically higher diffusion coefficients than the $\left[\mathrm{P}_{122 \mathrm{i} 4}\right]$ cation in the neat OIPC at all temperatures and diffusion times measured. The nanoconfined OIPC, on the other hand, showed constant diffusion coefficients with increasing diffusion time for both cation and anion, as shown in Fig. 6(c) and (d). This is because the size of the confined space of $40 \mathrm{~nm}$ is significantly smaller than the mean-square diffusion displacement $\sqrt{6 D_{0} t}$, which is estimated to be in the order of 1-5 $\mu \mathrm{m}$. Therefore, the observed diffusion coefficient reached an asymptotic value $D_{\infty}$. Interestingly, the cation and anions show very similar diffusion coefficients in the nanoconfined sample, which is in stark contrast with the neat OIPC where a significantly higher anion diffusion is observed in this study, as well as the previous experimental ${ }^{28}$ and MD simulation $^{38}$ studies. Note that the ionic diffusion in neat OIPC is time-dependent and the measured self-diffusion is underestimated because ions hit the walls of pores, thus direct comparison of the diffusion between neat and nanoconfined species is invalid. To obtain a time independent (free diffusion) coefficient, an extremely short diffusion time is required, which is not accessible by our instrument due to hardware limitations on the gradient strength as well as RF pulse lengths.

The Padé approximation has been used to fit the experimental data, ${ }^{39,40}$ and to interpolate between short time limit $\left(D_{0}\right)$ and the long-time asymptotic limit $D_{\infty}$. This method uses an adjustable fitting parameter, $\theta$, which has units of time and is expected to scale with the square of the pore size:

$$
\frac{D_{t}}{D_{0}}=1-\left(1-\frac{1}{\alpha}\right) \times \frac{\frac{4 \sqrt{D_{0} t}}{9 \sqrt{\pi}} \cdot \frac{S}{V}+\left(1-\frac{1}{\alpha}\right) \cdot \frac{t}{\theta}}{\left(1-\frac{1}{\alpha}\right)+\frac{4 \sqrt{D_{0} t}}{9 \sqrt{\pi}} \cdot \frac{S}{V}+\left(1-\frac{1}{\alpha}\right) \cdot \frac{t}{\theta}}
$$

where $\alpha$ is the tortuosity, $\frac{S}{V}$ is the surface to volume ratio of the confined space. Using this model to fit the experimental data shown in Fig. 6(a) and (b) allows us to estimate the free diffusion coefficients of cation and anion in neat OIPC, and the results are compared with the $40 \mathrm{~nm}$ confined OIPC in Fig. 7. Interestingly, at higher temperatures of $90 \& 80{ }^{\circ} \mathrm{C}$, the diffusion coefficients of $\left[\mathrm{PF}_{6}\right]$ anion and $\left[\mathrm{P}_{122 \mathrm{i} 4}\right]$ cation confined in the $40 \mathrm{~nm}$ pores, as well as the $\left[\mathrm{PF}_{6}\right]$ anion in neat OIPC are similar, whereas the $\left[\mathrm{P}_{122 \mathrm{i}}\right]$ cation of the neat OIPC shows notably lower diffusion. This result suggests that the cation diffusion, which was suppressed in neat OIPC, is enhanced in the nanoconfined sample, mostly likely due to the surface interactions between the ions and the $\mathrm{Al}_{2} \mathrm{O}_{3}$ walls. At lower temperatures of 50 and $60{ }^{\circ} \mathrm{C}$, while the error of fitting is large due to the poor signal-to-noise ratio, it may still be valid to

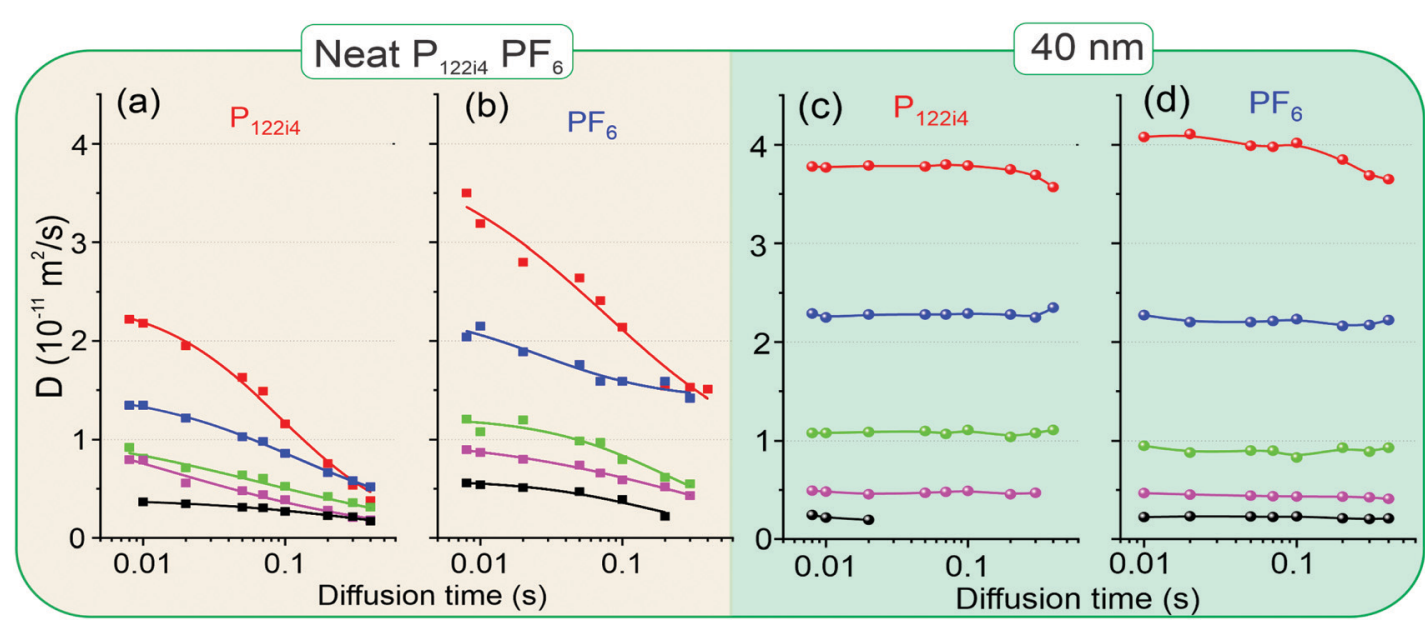

Fig. 6 Time-dependent diffusion coefficients of the neat and $40 \mathrm{~nm}$ confined OIPC measured at variable temperatures, from top to bottom, red $90{ }^{\circ} \mathrm{C}$, blue $80{ }^{\circ} \mathrm{C}$, green $70{ }^{\circ} \mathrm{C}$, purple $60^{\circ} \mathrm{C}$ and black $50{ }^{\circ} \mathrm{C}$. (a) $\left[\mathrm{P}_{122 i 4}\right]$ cation diffusion in neat OIPC; (b) $\left[\mathrm{PF}_{6}\right]$ anion diffusion in neat OIPC; (c) $\left[\mathrm{P}_{122 i 4}\right]$ cation diffusion in $40 \mathrm{~nm}$ sample; (d) $\left[\mathrm{PF}_{6}\right]$ anion diffusion in $40 \mathrm{~nm}$ sample. The dots represent experimental data points. The solid lines in (a) and (b) represent the best fit using Padé approximation (eqn (1)), whereas solid lines in (c) and (d) are drawn to guide the eyes only. To achieve a better reproducible thermal history, the temperatures were cooled down from 90 to $50{ }^{\circ} \mathrm{C}$ for all the experiments. 


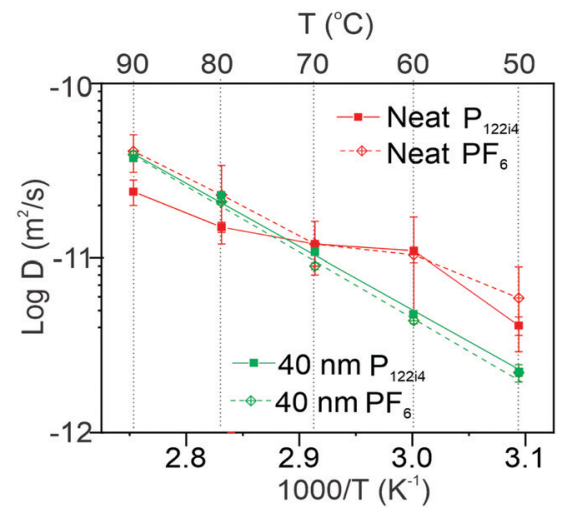

Fig. 7 Comparison of the diffusion coefficients of the extrapolated diffusion coefficient of neat OIPC $D_{0}$ to the $40 \mathrm{~nm}$ sample. The latter was obtained by a number averaging of the $D_{\mathrm{t}}$ values with different diffusion times.

claim that the difference between the two samples is significant relative to the error. A clear plateau in the temperature region of 70-60 ${ }^{\circ} \mathrm{C}$ was observed for the neat OIPC sample, which corresponds to the phase II to III transition of the OIPC, and this discontinuity is also observed in the Arrhenius plot of conductivity of neat OIPC in Fig. 2(c). The nanoconfined sample, however, showed a typical Arrhenius diffusion behaviour in the studied temperature range, and fitting of the experimental results revealed an activation energy of $70 \pm 4$ and $72 \pm 3 \mathrm{~kJ} \mathrm{~mol}^{-1}$ for the cation and anion, respectively.

It is worth noting that the diffusion coefficients of the $40 \mathrm{~nm}$ sample were measured in a powder sample where the nanochannels are randomly oriented in all possible directions. If the channels were aligned, which is the case for conductivity measurements, the obtained diffusion coefficient could be 3 times the observed diffusion coeffients shown in Fig. 6 and 7. The allignment dependency of the PFG-NMR measured diffusion coefficient due to the fact that the magnetic gradient is one dimensional: only the diffusion along the gradient direction is considered effective, whereas that perpendicular to the gradient is measured to be zero. ${ }^{41,42}$ This would mean systematically higher diffusion coefficients for both cation and anion in the nano-confined ion channels compared to the neat OIPC, and this enhancement is a direct consequence of using the nanoconfined through pores. As discussed earlier, the conductivity enhancement at lower temperatures was way beyond 3 fold. At $30{ }^{\circ} \mathrm{C}$ for example, the enhancement was 44 times. This means that the alignment effect was not the only factor that contributes to the conductivity, other reasons such as enhancement in the population of mobile species, as evident from the static ${ }^{1} \mathrm{H}$ NMR spectra in Fig. 4a, must have played a role. At $120^{\circ} \mathrm{C}$, on the other hand, the enhancement was 5 times, suggesting that the alignment of conduction pathways was one of the major factors that contribute to the ionic conductivity enhancements.

\section{Conclusions}

In this study, we investigated the effect of nano-confinement on the ion conduction, rotational dynamics and translational motions (diffusion) of an organic ionic plastic crystal (OIPC) material, $\left[\mathrm{P}_{122 \mathrm{i} 4}\right]\left[\mathrm{PF}_{6}\right]$ in mesoporous $\mathrm{Al}_{2} \mathrm{O}_{3}$ membranes with hexagonally aligned through pores. Our multi-nuclear static NMR lineshape analysis suggested a highly decoupled cation and anion dynamics throughout the entire solid phases of $\left[\mathrm{P}_{122 i 4}\right]\left[\mathrm{PF}_{6}\right]$, and each solid-solid transition is primarily associated with onset of motions of one ion species. The phase IV $\rightarrow$ III transition at $26^{\circ} \mathrm{C}$, and phase II $\rightarrow$ I transition at $120{ }^{\circ} \mathrm{C}$ are mainly associated with the onset of motions of cation, whereas the phase III $\rightarrow$ II transition at $72{ }^{\circ} \mathrm{C}$ is associated with that of the anion. The nano-confinement affects the populations and dynamics of the mobile ions located in the amorphous or defect regions only, while leaving the crystalline regions less affected. The nanoconfined OIPC was found to exhibit 44 times higher ionic conductivity at $30^{\circ} \mathrm{C}$, and this was attributed to the combination of (1) alignment of ionic conduction pathways, and (2) significantly higher population of mobile species. At higher temperatures above $120^{\circ} \mathrm{C}$ where all the ionic species become mobile, the alignment of conduction pathways becomes one of the major factors that contributes to the ionic conductivity enhancements. Last but not the least, the mesoporous $\mathrm{Al}_{2} \mathrm{O}_{3}$ substrate was found to selectively change the rotation and translational motion of $\left[\mathrm{P}_{122 i 4}\right]$ cation, whereas leaving that of the $\left[\mathrm{PF}_{6}\right]$ anion less affected.

\section{Conflicts of interest}

There are no conflicts to declare.

\section{Acknowledgements}

M. F. thanks the Ikerbasque foundation for a visiting professorial fellowship. The authors acknowledge the Australian Research Council for funding through CE140100012. Deakin University's advanced characterisation facility is acknowledged for use of the NMR instruments, funded through the ARC grant LE110100141. J. M. thanks MCIU for the Ramón y Cajal contract and the grant Ref. PGC2018-094620-A-I00. Authors would like to thank the financial support provided by the IONBIKE RISE project. This project has received funding from the European Union's Horizon 2020 research and innovation programme under the Marie Skłodowska-Curie grant agreement No. 823989.

\section{References}

1 K. A. Mauritz and R. B. Moore, State of Understanding of Nafion, Chem. Rev., 2004, 104(10), 4535-4586.

2 D. Mecerreyes, Polymeric ionic liquids: Broadening the properties and applications of polyelectrolytes, Prog. Polym. Sci., 2011, 36(12), 1629-1648.

3 X. Wang, H. Zhu, G. W. Greene, Y. Zhou, M. YoshizawaFujita, Y. Miyachi, M. Armand, M. Forsyth, J. M. Pringle and P. C. Howlett, Organic Ionic Plastic Crystal-Based Composite Electrolyte with Surface Enhanced Ion Transport and Its 
Use in All-Solid-State Lithium Batteries, Adv. Mater. Technol., 2017, 2(7), 1700046.

4 L. Jin, P. C. Howlett, J. M. Pringle, J. Janikowski, M. Armand, D. R. MacFarlane and M. Forsyth, An organic ionic plastic crystal electrolyte for rate capability and stability of ambient temperature lithium batteries, Energy Environ. Sci., 2014, 7(10), 3352-3361.

5 M. Díaz, A. Ortiz, J. M. Pringle, X. Wang, R. Vijayaraghavan, D. R. MacFarlane, M. Forsyth and I. Ortiz, Protic plastic crystal/PVDF composite membranes for Proton Exchange Membrane Fuel Cells under non-humidified conditions, Electrochim. Acta, 2017, 247, 970-976.

6 J. Luo, A. H. Jensen, N. R. Brooks, J. Sniekers, M. Knipper, D. Aili, Q. Li, B. Vanroy, M. Wübbenhorst, F. Yan, L. Van Meervelt, Z. Shao, J. Fang, Z.-H. Luo, D. E. De Vos, K. Binnemans and J. Fransaer, 1,2,4-Triazolium perfluorobutanesulfonate as an archetypal pure protic organic ionic plastic crystal electrolyte for all-solid-state fuel cells, Energy Environ. Sci., 2015, 8(4), 1276-1291.

7 A. Lennert, K. Wagner, R. Yunis, J. M. Pringle, D. M. Guldi and D. L. Officer, Efficient and Stable Solid State Dye Sensitized Solar Cells by Combination of Phosphonium Organic Ionic Plastic Crystals with Silica, ACS Appl. Mater. Interfaces, 2018, 10, 32271-32280, DOI: 10.1021/acsami.8b12334.

8 C. Shi, L. Qiu, X. Chen, H. Zhang, L. Wang and F. Yan, Silica Nanoparticle Doped Organic Ionic Plastic Crystal Electrolytes for Highly Efficient Solid-State Dye-Sensitized Solar Cells, ACS Appl. Mater. Interfaces, 2013, 5(4), 1453-1459.

9 D. Hwang, D. Y. Kim, S. M. Jo, V. Armel, D. R. MacFarlane, D. Kim and S.-Y. Jang, Highly Efficient Plastic Crystal Ionic Conductors for Solid-state Dye-sensitized Solar Cells, Sci. Rep., 2013, 3, 3520.

10 H. Zhu, D. R. MacFarlane, J. M. Pringle and M. Forsyth, Organic Ionic Plastic Crystals as Solid-State Electrolytes, Trends Chem., 2019, 1, 1.

11 F. Makhlooghiazad, D. Gunzelmann, M. Hilder, D. R. MacFarlane, M. Armand, P. C. Howlett and M. Forsyth, Mixed Phase Solid-State Plastic Crystal Electrolytes Based on a Phosphonium Cation for Sodium Devices, Adv. Energy Mater., 2017, 7(2), 1601272.

12 H. Zhu, U. a. Rana, V. Ranganathan, L. Jin, L. A. O’Dell, D. R. MacFarlane and M. Forsyth, Proton transport behaviour and molecular dynamics in the guanidinium triflate solid and its mixtures with triflic acid, J. Mater. Chem. A, 2014, 2(3), 681-691.

13 D. R. MacFarlane and M. Forsyth, Plastic crystal electrolyte materials: new perspectives on solid state ionics, Adv. Mater., 2001, 13(12-13), 957-966.

14 H. Ishida, T. Iwachido, N. Hayama, R. Ikeda, M. Terashima and D. Nakamura, Self-diffusion and reorientation of methylammonium ions in $\left(\mathrm{CH}_{3} \mathrm{NH}_{3}\right)_{2} \mathrm{ZnCl}_{4}$ Crystals as studied by ${ }^{1}$ H-NMR, Z. Naturforsch., A: Phys. Sci., 1989, 44, 741-746.

15 H. Ishida, R. Ikeda and D. Nakamura, ${ }^{1} \mathrm{H}$ NMR Studies on the Reorientational Motions of Cations in Four Solid Phases of Methylammonium Iodide and the Self-Diffusion of Ions in Its Highest-Temperature Solid Phase, Bull. Chem. Soc. Jpn., 1986, 59(3), 915-924.

16 A. Basile, M. Hilder, F. Makhlooghiazad, C. Pozo-Gonzalo, D. R. MacFarlane, P. C. Howlett and M. Forsyth, Ionic Liquids and Organic Ionic Plastic Crystals: Advanced Electrolytes for Safer High Performance Sodium Energy Storage Technologies, Adv. Energy Mater., 2018, 8(17), 1703491.

17 J. Rao, R. Vijayaraghavan, X. Wang, Y. Zhou, P. C. Howlett, D. R. MacFarlane, M. Forsyth and H. Zhu, Influence of Electrospun Poly(vinylidene difluoride) Nanofiber Matrix on the Ion Dynamics of a Protic Organic Ionic Plastic Crystal, J. Phys. Chem. C, 2018, 122(26), 14546-14553.

18 X. Wang, H. Zhu, Y. Zhou, M. Yoshizawa-Fujita, Y. Miyachi, M. Armand, M. Forsyth, J. M. Pringle and P. C. Howlett, Organic Ionic Plastic Crystal-Based Composite Electrolyte with Surface Enhanced Ion Transport and Its Use in AllSolid-State Lithium Batteries, Adv. Mater. Technol., 2017, 2(7), 1700046.

19 J. Efthimiadis, G. J. Annat, J. Efthimiadis, M. Forsyth and D. R. MacFarlane, Solid state ion transport and phase behaviour in composites of $\mathrm{N}, \mathrm{N}$-methyl propylpyrrolidinium tetrafluoroborate and amorphous polyethylene oxide, Phys. Chem. Chem. Phys., 2003, 5(24), 5558-5564.

20 N. Goujon, R. Kerr, C. Gervillié, Y. V. Oza, L. A. O’Dell, P. C. Howlett and M. Forsyth, Macrophase-Separated Organic Ionic Plastic Crystals/PAMPS-Based Ionomer Electrolyte: A New Design Perspective for Flexible and Highly Conductive Solid-State Electrolytes, ACS Omega, 2020, 5(6), 2931-2938.

21 X. Wang, G. M. A. Girard, H. Zhu, R. Yunis, D. R. MacFarlane, D. Mecerreyes, A. J. Bhattacharyya, P. C. Howlett and M. Forsyth, Poly(ionic liquid)s/Electrospun Nanofiber Composite Polymer Electrolytes for High Energy Density and Safe Li Metal Batteries, ACS Appl. Energy Mater., 2019, 2(9), 6237-6245.

22 J. Adebahr, N. Ciccosillo, Y. Shekibi, D. R. MacFarlane, A. J. Hill and M. Forsyth, The "filler-effect" in organic ionic plastic crystals: Enhanced conductivity by the addition of nano-sized $\mathrm{TiO}_{2}$, Solid State Ionics, 2006, 177(9), 827-831.

23 J. M. Pringle, Y. Shekibi, D. R. MacFarlane and M. Forsyth, The influence of different nanoparticles on a range of organic ionic plastic crystals, Electrochim. Acta, 2010, 55(28), 8847-8854.

24 Y. Shekibi, S. J. Pas, N. M. Rocher, B. R. Clare, A. J. Hill, D. R. MacFarlane and M. Forsyth, Surprising effect of nanoparticle inclusion on ion conductivity in a lithium doped organic ionic plastic crystal, J. Mater. Chem., 2009, 19(11), 1635-1642.

25 X. Wang, H. Zhu, G. W. Greene, J. Li, N. Iranipour, C. Garnier, J. Fang, M. Armand, M. Forsyth, J. M. Pringle and P. C. Howlett, Enhancement of ion dynamics in organic ionic plastic crystal/PVDF composite electrolytes prepared by co-electrospinning, J. Mater. Chem. A, 2016, 4(25), 9873-9880.

26 Y. Shekibi, A. Gray-Weale, D. R. MacFarlane, A. J. Hill and M. Forsyth, Nanoparticle Enhanced Conductivity in Organic 
Ionic Plastic Crystals: Space Charge versus Strain Induced Defect Mechanism, J. Phys. Chem. C, 2007, 111(30), 11463-11468.

27 M. Forsyth, F. Chen, L. A. O'Dell and K. Romanenko, New insights into ordering and dynamics in organic ionic plastic crystal electrolytes, Solid State Ionics, 2016, 288, 160-166.

28 K. Romanenko, L. Jin, L. A. Madsen, J. M. Pringle, L. A. O'Dell and M. Forsyth, Anisotropic MRI Contrast Reveals Enhanced Ionic Transport in Plastic Crystals, J. Am. Chem. Soc., 2014, 136(44), 15638-15645.

29 F. Chen, L. Jin, S. W. de Leeuw, J. M. Pringle and M. Forsyth, Atomistic simulation of structure and dynamics of the plastic crystal diethyl(methyl)(isobutyl)phosphonium hexafluorophosphate, J. Chem. Phys., 2013, 138(24), 244503.

30 L. Jin, K. M. Nairn, C. M. Forsyth, A. J. Seeber, D. R. MacFarlane, P. C. Howlett, M. Forsyth and J. M. Pringle, Structure and Transport Properties of a Plastic Crystal Ion Conductor: Diethyl(methyl)(isobutyl)phosphonium Hexafluorophosphate, J. Am. Chem. Soc., 2012, 134(23), 9688-9697.

31 A. Bielecki and D. P. Burum, Temperature Dependence of ${ }^{207} \mathrm{~Pb}$ MAS Spectra of Solid Lead Nitrate. An Accurate, Sensitive Thermometer for Variable-Temperature MAS, J. Magn. Reson., Ser. A, 1995, 116(2), 215-220.

32 A. L. Van Geet, Calibration of methanol nuclear magnetic resonance thermometer at low temperature, Anal. Chem., 1970, 42(6), 679-680.

33 N. Bloembergen, E. M. Purcell and R. V. Pound, Relaxation Effects in Nuclear Magnetic Resonance Absorption, Phys. Rev., 1948, 73(7), 679-712.

34 K. Romanenko, J. M. Pringle, L. A. O'Dell and M. Forsyth, New insights into the thermal behaviour of organic ionic plastic crystals: magnetic resonance imaging of polycrystalline morphology alterations induced by solid-solid phase transitions, Phys. Chem. Chem. Phys., 2015, 17(29), 18991-19000.

35 P. N. Sen, L. M. Schwartz, P. P. Mitra and B. I. Halperin, Surface relaxation and the long-time diffusion coefficient in porous media: Periodic geometries, Phys. Rev. B: Condens. Matter Mater. Phys., 1994, 49(1), 215-225.

36 L. L. Latour, P. P. Mitra, R. L. Kleinberg and C. H. Sotak, Time-Dependent Diffusion Coefficient of Fluids in Porous Media as a Probe of Surface-to-Volume Ratio, J. Magn. Reson., Ser. A, 1993, 101(3), 342-346.

37 T. Bickel, A note on confined diffusion, Phys. A, 2007, 377(1), 24-32.

38 F. Chen, L. Jin, S. W. D. Leeuw, J. M. Pringle and M. Forsyth, Atomistic simulation of structure and dynamics of the plastic crystal diethyl(methyl)(isobutyl)phosphonium hexafluorophosphate, J. Chem. Phys., 2013, 138(24), 244503.

39 P. P. Mitra, P. N. Sen and L. M. Schwartz, Short-time behavior of the diffusion coefficient as a geometrical probe of porous media, Phys. Rev. B: Condens. Matter Mater. Phys., 1993, 47(14), 8565-8574.

40 P. P. Mitra, P. N. Sen, L. M. Schwartz and P. Le Doussal, Diffusion propagator as a probe of the structure of porous media, Phys. Rev. Lett., 1992, 68(24), 3555-3558.

41 J. Li, K. G. Wilmsmeyer and L. A. Madsen, Anisotropic Diffusion and Morphology in Perfluorosulfonate Ionomers Investigated by NMR, Macromolecules, 2009, 42(1), 255-262.

42 J. Li, K. G. Wilmsmeyer and L. A. Madsen, Hydrophilic Channel Alignment Modes in Perfluorosulfonate Ionomers: Implications for Proton Transport, Macromolecules, 2008, 41(13), 4555-4557. 